\title{
ON $q$-POWER CYCLES IN CUBIC GRAPHS
}

\author{
JULIEN Bensmail \\ Department of Applied Mathematics and Computer Science \\ Technical University of Denmark \\ DK-2800 Lyngby, Denmark \\ e-mail: julien.bensmail.phd@gmail.com
}

\begin{abstract}
In the context of a conjecture of Erdős and Gyárfás, we consider, for any $q \geq 2$, the existence of $q$-power cycles (i.e., with length a power of $q$ ) in cubic graphs. We exhibit constructions showing that, for every $q \geq 3$, there exist arbitrarily large cubic graphs with no $q$-power cycles. Concerning the remaining case $q=2$ (which corresponds to the conjecture of Erdös and Gyárfás), we show that there exist arbitrarily large cubic graphs whose all 2-power cycles have length 4 only, or 8 only.
\end{abstract}

Keywords: cubic graphs, $q$-power cycles, Erdős-Gyárfás conjecture.

2010 Mathematics Subject Classification: 68R10, 05C38.

\section{REFERENCES}

[1] P. Erdős, Some old and new problems in various branches of combinatorics, Discrete Math. 165/166 (1997) 227-231. doi:10.1016/S0012-365X(96)00173-2

[2] D. Daniel and S.E. Shauger, A result on the Erdös-Gyárfás conjecture in planar graphs, Congr. Numer. 153 (2001) 129-139.

[3] C.C. Heckman and R. Krakovski, Erdös-Gyárfás conjecture for cubic planar graphs, Electron. J. Combin. 20 (2013) \#P7.

[4] K. Markström, Extremal graphs for some problems on cycles in graphs, Congr. Numer. 171 (2004) 179-192.

[5] P.S. Nowbandegani, H. Esfandiari, M.H.S. Haghighi and K. Bibak, On the ErdösGyárfás conjecture in claw-free graphs, Discuss. Math. Graph Theory 34 (2014) 635-640. doi:10.7151/dmgt.1732 
[6] S.E. Shauger, Results on the Erdös-Gyárfás conjecture in $K_{1, m}$-free graphs, Congr. Numer. 134 (1998) 61-65.

[7] D. West, Erdôs-Gyárfás conjecture on 2-power cycle lengths, Open ProblemsGraph Theory and Combinatorics.

http://www.math.illinois.edu/ dwest/openp/2powcyc.html

Received 3 October 2015

Revised 18 March 2016

Accepted 30 March 2016 